\title{
Unilateral drainage of bilateral chronic subdural hematoma
}

\begin{abstract}
Bilateral chronic subdural hematoma (bCSDH) is a condition frequently encountered in neurosurgical practice, and it is usually the result of head trauma. Despite its frequency, no consensus currently exists regarding its optimal treatment. While the use of corticosteroids in the treatment of chronic subdural hematoma is not currently accepted by the neurosurgical community, there is enough evidence in the literature that supports its use. In bCSDH the unilateral burr hole evacuation of the larger of the subdural collections followed by a course of corticosteroids seems to be an effective and safer course of action in the management of bilateral chronic subdural hematoma.
\end{abstract}

Keywords: subdural hematoma, head trauma, corticosteroids, craniostomy
Volume II Issue 6 - 202 I

\author{
Allan J Drapkin,' Manuel Campos $\mathrm{P}^{2}$ \\ 'Department of Neurosurgery, Rutgers Biomedical and Health \\ Sciences, USA \\ ${ }^{2}$ Department of Neurosurgery, Clinic Las Condes, Chile
}

Correspondence: Allan J Drapkin, Alejandro Serani Norte 9458. Depto. 402,Vitacura. Santiago. Chile. Postal code 764080I, Tel +56233082519, Email ajdrapkin@gmail.com

Received: November 10, 2021 | Published: November 23, 2021

\section{Introduction}

Unilateral chronic subdural hematoma (uCSDH) is a condition frequently encountered in neurosurgical practice, a frequency that fluctuates between 5.3 and 13.5 cases per 100.000 persons/year and increases in the elderly population. ${ }^{1,2}$ It will continue to grow because of the increasing age of the general population and the frequent use of anticoagulants and antiplatelet medications.

The currently accepted treatment for uCSDH is twist-drill or burrhole craniostomy with evacuation of the subdural collection with or without irrigation of the subdural space and the elective placement of a closed-system subdural drainage. ${ }^{3}$ Despite its surgical simplicity, this procedure is not bereft of complications. ${ }^{4}$ bCSDH is also not rare, and presents a significant rate of complications and recurrences. Despite this situation, no well-defined treatment plan for it has been established to date, and its optimal management remains a matter of debate. ${ }^{5}$ Such a case is presented herewith with the treatment variation we implemented.

\section{Method}

A review of the literature under the heading of chronic subdural hematoma was done and the reference lists of the publications thus gathered was reviewed in order to augment the data. Moreover, our case of a bilateral chronic subdural hematoma and its treatment is herewith presented.

\section{Results}

Although the use of corticosteroids in the treatment of chronic subdural hematoma is not currently accepted by the neurosurgical community, there is enough evidence in the literature that supports its use.

\section{Case report}

An elderly patient, with a past medical history of well controlled hypertension, and a daily dose of $81 \mathrm{mg}$ of Aspirin, experienced, for the three months preceding presentation, generalized headaches which gradually increased in frequency and intensity. In the last week the headaches became associated with memory disturbances and mental confusion which prompted her consultation. Her physical examination detected no obvious neurological deficits but an emergency CT scan revealed a bilateral chronic subdural hematoma (Figure 1A), larger on the right side with midline shift (Figure 1B) for which she was admitted.

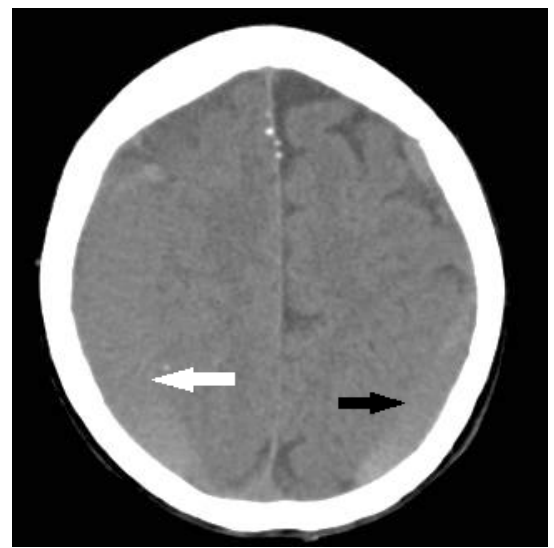

Figure IA Non-contrasted CT scan showing a large, mostly isodense, right sided chronic subdural hematoma (white arrow) and a smaller left subdural collection (black arrow).

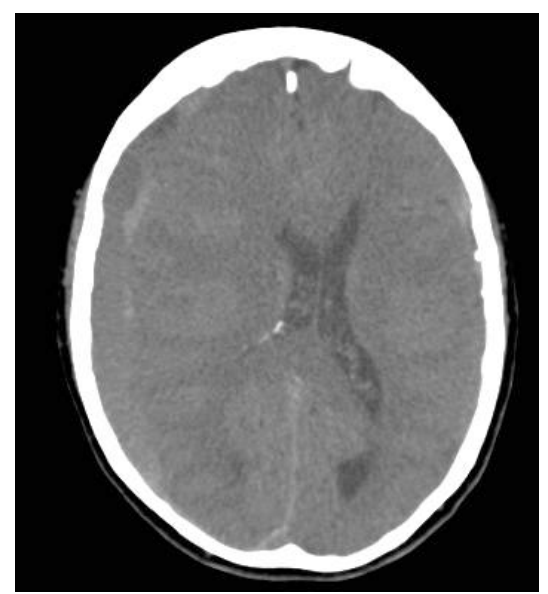

Figure I B Non-contrasted CT scan showing marked left sided midline shift.

Routine blood tests, a chest $\mathrm{x}$-ray and an EKG revealed no significant abnormalities. The patient was subsequently brought to the operating 
room where, under general endotracheal anesthesia and in the supine position with some head elevation, burr-holes were made in the right posterior frontal and parietal areas overlying the largest of the subdural collections. The dura was opened in a cruciform fashion at both sites and each one of the resulting dural quadrants was shrunk by bipolar coagulation so as to prevent the creation of a ball-valve mechanism that could interfere with the egress of the subdural collection into the subgaleal space where it could be absorbed. Moreover, the subdural space was irrigated with physiological solution but no subdural drain was placed. The patient tolerated the procedure well. The following day a repeat CT scan was done to assess the postoperative condition (Figure 2). She was then started on a ten-day course of Prednisone 5 $\mathrm{mg}$ PO QID and she was discharged. A repeat CT scan was done three weeks later (Figure 3).

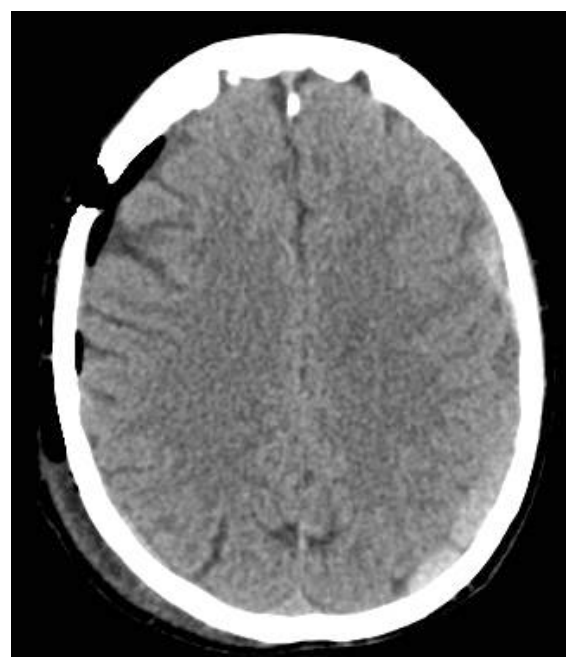

Figure 2 Non-contrasted CT scan showing clearance of the preexisting right sided chronic subdural hematoma, a right frontal burr hole with a small postoperative pneumocephalus with persistence of the smaller left sided subdural hematoma.

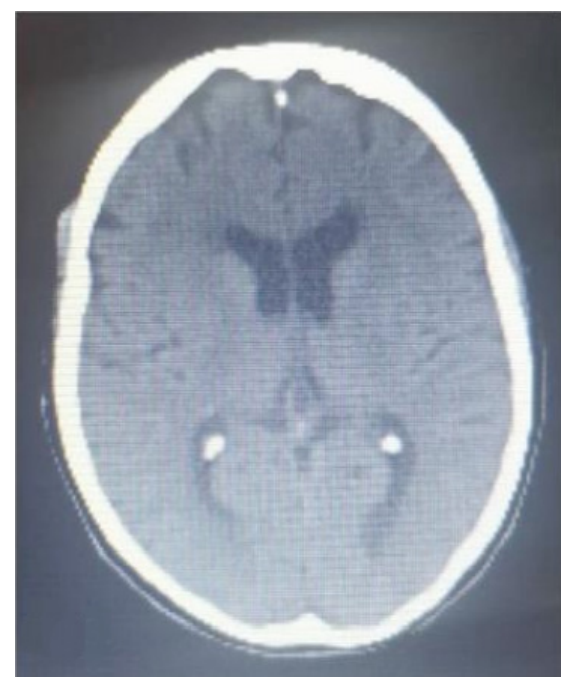

Figure 3 Non-contrasted CT scan showing clearance of both subdural collections.

\section{Discussion}

The recognized cause of CSDH is usually a trivial head trauma that causes acceleration/deceleration of the intracranial content resulting in a traumatic cleavage between the inner layer of the duramater and the arachnoid thus transforming a virtual space into an actual subdural space. ${ }^{6}$ The simultaneous tear of the bridging veins in that area causes bleeding into that subdural space, a mechanism whose anatomical basis has been clearly demonstrated. ${ }^{7}$ The symptomatic expression of this subdural hematoma usually lags by weeks or months and, because the original trauma is usually a minor one, by the time of presentation it is frequently forgotten by the patients, a fact that explains the 30 $50 \%$ of cases lacking such historical data upon admission. ${ }^{2}$

Blood within the subdural space incite an inflammatory reaction with deposition of fibrin and the eventual formation of subdural membranes that encapsulates the subdural blood. These neomembranes, particularly the outer one, possess high levels of plasminogen and plasminogen activator ${ }^{8}$ and several angiogenesispromoting factors, such as the vascular endothelial growth factor (VEGF), which induces the formation, in these neo membranes, of immature and leaky blood vessels with large gap junctions and weak basement membranes. ${ }^{9}$ The high content of plasminogen and plasminogen activator existing in the neomembranes, easily diffuses into the subdural collection, causing the enzymatic fibrinolysis and liquefaction of the subdural clot, resulting in a hyperfibrinolytic state with a high level of fibrin degradation products. This brings about the formation of defective clots that are easily lysed. These, together with the leaky blood vessels created in the outer neo membrane, result in frequent effusions of plasma and/or bleeding into the subdural collection leading to its gradual increase in size. ${ }^{10}$

The goal of any medical or surgical treatment for chronic subdural hematoma should be the complete resolution of the subdural collection, allowing brain re-expansion and the apposition of the outer subdural membrane onto the inner one, hypothetically causing scarring between these two membranes thus sealing the subdural space initially created and preventing the development of any further subdural collection, at least in the affected area. Confirmation of this hypothesis would not be easy to accomplish since it would require the pathological study of an autopsy series of difficult to collect cases that have recovered from CSDH but died from other causes.

Medical treatment of CSDH have been attempted with the use of Tranexamic acid, because of its antifibrinolytic activity, ${ }^{11}$ as well as with the angiotensin-converting enzyme (ACE) because of its capacity to inhibit angiogenesis. ${ }^{12}$ While the results obtained with these two pharmacological modalities have been encouraging, more experience with their use in this setting is needed before these could be incorporated into the $\mathrm{CSDH}$ treatment paradigm.

Corticosteroids have also been suggested and used for the treatment of CSDH in cases where anesthesia and surgery are not feasible,${ }^{13}$ for cases with mild or moderate symptomatology, ${ }^{14}$ as an adjuvant to the surgical procedure ${ }^{16,17}$ and for CSDH too small to warrant surgery, ${ }^{16}$ while others have suggested its use as a first line of treatment for $\mathrm{CSDH} .{ }^{17}$

While Dexamethasone's therapeutic effect on CSDH is still debated, ${ }^{18}$ previous experience ${ }^{16,17}$ supports its use considering that corticosteroids actions interfere with the three main processes involved in the genesis and persistence of CSDH. Namely its antiinflammatory activity, ${ }^{19}$ its inhibition of plasminogen activator thus reducing fibrinolysis ${ }^{20}$ and its inhibition of VEGF, thus decreasing angiogenesis, ${ }^{21}$ all actions that should promote $\mathrm{CSDH}$ resolution.

While the use of Dexamethasone in small CSDH is very effective without the need for surgical intervention, ${ }^{16}$ the sole use of Dexamethasone in large CSDH causing mass effect and midline shift, is not advisable because it would require a prolonged treatment with 
relative high doses of the drug that could result in significant sideeffects. Furthermore, an emergent surgical evacuation could become necessary during this prolonged course of treatment. ${ }^{14,18}$ While the use of corticosteroids as the sole treatment of CSDH has demonstrated its capacity to reduce the rate of recurrences in $\mathrm{CSDH}$, this type of treatment resulted in an increased rate of side-effects. ${ }^{18}$ This fact notwithstanding, the use of corticosteroids but as an adjuvant to the surgical evacuation of CSDH and using a lesser dose than the one used in that study has proven very effective and safe..$^{16,17}$

bCSDH represent between 16 and $24 \%$ of the observed cases of $\mathrm{CSDH}^{22,23}$ This bilateral form shows less lateralizing neurological deficits and less radiological midline shift than its unilateral counterpart, facts that may lead to a delay in its diagnosis. ${ }^{24}$ Moreover, bCSDH presents a reported $28 \%$ rate of recurrence, compared with the rate of recurrence in the uCSDH which is $9.59 \%$.

Two recently published articles studying the results of unilateral surgical evacuation in $\mathrm{bCSDH}^{25,26}$ comprising a total of 394 cases stated that currently the decision to perform a unilateral evacuation on bCSDH is based on the relative volume of each subdural collection and in the presence of lateralizing clinical signs. The unilateral procedure in bCSDH was found in these studies, to be an independent risk factor for reoperation, a fact that led these authors to recommend the bilateral evacuation from the start. This recommendation cannot be taken lightly because, aside from requiring a longer time of anesthesia/ sedation and increased intraoperative manipulation, the simultaneous evacuation of both CSDH in the geriatric population, where an associated brain atrophy may exist, ${ }^{27}$ could retard the postoperative brain expansion, and lead to intracranial hypotension with the potential development of tension pneumocephalus. ${ }^{28}$ An additional recent study analyzing this situation, ${ }^{29}$ established that the hypodense and the homogeneous CT densities in the preoperative non-operated subdural hematoma as well as the volume of the postoperative nonoperated subdural collection, were important factors in predicting a postoperative contralateral hematoma progression, which could require a second surgical intervention.

The case presented herewith suggest a less invasive alternative by combining the unilateral evacuation of the larger subdural collection, causing a rapid improvement in the preoperative symptoms, followed by a ten days postoperative course of corticosteroids, which, by using a moderate dosage, should provoke no mayor side effects. The only additional requirement this regimen would impose is the close monitoring and control of the blood sugar level in those clinical or subclinical diabetic patients, for a period of time not significantly different than the usual period of postoperative follow up.

\section{Conclusion}

The potential risk inherent to the unilateral evacuation of a bCSDH, can be avoided with the postoperative use of corticosteroids which would promote the gradual and complete resolution of both subdural collections thus decreasing the expense and time involved in a more prolonged follow-up period with serial repeated CT scans. Obviously, the efficacy of this method should be confirmed by a multicenter randomized controlled study.

\section{Author contributions}

AJD contributed with the original conception of the work, reviewed the related literature, and wrote the first draft. MCP evaluated and operated the patient herewith presented, participated in the surgical planning, gathered the radiological material and critically reviewed the first draft. Both authors read the final version of the manuscript, approved its submission and are accountable for all aspects of the work.

\section{Data availability statement}

No data sets were generated or analyzed for this study.

\section{Acknowledgments}

The authors wish to thank Paola Vermeer PhD, Ms. Camila Berner M.Sc. and Ms. Elizabeth Gonzalez for their bibliographical and technical assistance in the performance of this work.

\section{Conflicts of interest}

The authors declare that they have no conflicts of interest.

\section{Ethics statement}

Written informed consent was obtained from the individual prior to the publication of any potentially identifiable images or data included in this article.

\section{References}

1. Kudo H, Kawamura K, Izawa I, et al. Chronic subdural hematoma in elderly people. Present status in Awaji Island epidemiologic prospect. Neuro Med chir (Tokyo). 1992;32:207-209.

2. Adhiyaman V, Asghar M, Ganeshram KN, et al. Chronic subdural hematoma in the elderly. Postgrad Med J. 2002;78:71-75.

3. Santarius T, Kirkpatrick PJ, Ganesan D, et al. Use of drains versus no drains after burr-hole evacuation of chronic subdural hematoma: a randomized controlled trial. Lancet. 2009;374:1067-1073.

4. Gelabert-Gonzalez M, Iglesias-Pais, Garcia-Allut A, et al. Chronic subdural hematomas: surgical treatment and outcome in 1000 cases. Clin Neurol Neurosurg. 2005;107:223-229.

5. Agawa Y, MineHaru Y, Tani S, et al. Bilateral chronic subdural hematoma is associated with rapid progression and poor clinical outcome. Neurol Med (Tokyo). 2016;56:198-203.

6. Haines D, Louis HH, Al-Mefty O. The "subdural" space: A new look at an outdated concept. Neurosurgery. 1993;32(1):111-120.

7. Yamashima T, Friede RL. Why do bridging veins rupture into the virtual subdural space? J. Neurol Neurosurg Psychiat. 1984;47(2):121-127.

8. Ito H, Komai T, Yamamoto S. Fibrinolytic enzyme in the lining walls of chronic subdural hematoma. J Neurosurg. 1978;48:197-200.

9. Yamashima T, Yamamoto S, Friede RL. The role of endothelial gap junctions in the enlargement of chronic subdural hematomas. $J$ Neurosurg.1983;59(2):298-303.

10. Labadie EL, Glover D. Local alterations of hemostatic-fibrinolytic mechanisms reforming subdural hematomas. Neurology. 1975;25(7):669 675.

11. Kageyama H, Toyooka T, Tsuzuki N, et al Nonsurgical treatment of chronic subdural hematoma with Tranexamic acid. J Neurosurg. 2013;119:332-337.

12. Weigel R, Hohenstein A, Schlickum L, et al. Angiotensin converting enzyme inhibition for arterial hypertension reduces the risk of recurrence in patients with chronic subdural hematoma possibly by an antiangiogenic mechanism. Neurosurger. 2007;61(4):788-792.

13. Rudiger A, Ronsdorf A, Merlo A, et al. Dexamethasone treatment of a patient with large bilateral chronic subdural hematomata. Swiss Med Wkly. 2001;131:387. 
14. Bender MB, Christoff N. Nonsurgical treatment of subdural hematomas Arch Neurol. 1974;31(2):73-79.

15. Sun TF, Boet R, Poon WS, et al. Non-surgical primary treatment of chronic subdural hematoma: preliminary results of using dexamethasone. Brit J Neurosurg. 2005;19:327-333.

16. Drapkin AJ. Chronic subdural hematoma: patho-physiological basis for treatment. Brit J Neurosurg. 1991;5:467-473.

17. Delgado-López PD, Martín-Velasco V, Castilla-Diez JM, et al. Dexamethasone treatment in chronic subdural hematoma. Neurocirugía. 2009;20:346-359.

18. Hutchinson PJ, Edlmann E, Bulters D, et al. Trial of Dexamethasone for Chronic subdural hematoma. N Eng J Med. 2020;383:2616-2627.

19. Barnes PJ. How corticosteroid control inflammation; Quintiles Prize Lecture. Brit J Pharmacol. 2006;148:245-254.

20. Oikarinen A, Höyhtyä M, Järvinen M. Dexamethasone-induced plasminogen activator inhibitor: characterization, purification and preparation of monoclonal antibodies. Arch Dermatol Res. 1990;282(3):153-158.

21. Hegerman MA, Hennus MP, Kavelaars A, et al. Dexamethasone attenuates VEGF expression and inflammation but not barrier dysfunction in a murine model of ventilator-induced lung injury. Plos One. 2013;(2): e7374.

22. Berghauser Pont, Dammers R, Schouten JW, et al. Clinical factors associated with outcome in chronic subdural hematoma:a retrospective cohort study of patients on preoperative corticosteroid therapy. Neurosurgery. 2012;70:873-880.
23. MacFarlane MR, Weerakkody Y, Kathiravel Y. Chronic subdural hematomas are more common on the left than on the right. J Clin Neurosci. 2009;16:642-644.

24. Huang YH, Yang KY, Lee TC. Bilateral chronic subdural hematoma: what is the clinical significance? International J Surg. 2013;11:544-548.

25. Andersen-Ramberg NC, Poulsen FR, Bergholt B, et al. Bilateral subdural hematoma: unilateral or bilateral drainage? J Neurosurg. 2017;126:19051911.

26. Scheichel F, Popadic B, Ungerboeck K, et al. Contralateral progression after unilateral evacuation of bilateral chronic subdural hematomas; the volume relation ratio as prognostic factor? J Neurosurg. 2019;131:12271234.

27. Yang IL, Balser DS, Mikheev A, et al. Cerebral atrophy associated with development of chronic subdural hematoma. Brain Injury. 2012;26(1314):1731-1736.

28. Lavano A, Benvenuti D, Volpentesta G, et al. Symptomatic tension pneumocephalus after evacuation of chronic subdural hematoma: report of seven cases. Clin Neurol Neurosurg. 1990;92:35-41.

29. Shen J, Shao X, Gao Y, et al. Risk factors for contralateral hematoma progression after unilateral evacuation of bilateral chronic subdural hematomas. World Neurosurgery. 2019;126:e773-e778. 